\title{
Evolución de las escuelas de primeras letras y su impulso por Carlos III. Especial referencia al reglamento para el establecimiento de escuelas gratuitas para niñas de 1783
}

Evolution of the schools of first letters and its impulse by Carlos III. Special reference to the regulation for the establishment of free schools for girls of 1783

Dr. José Enrique Anguita Osuna Universidad Rey Juan Carlos -España joseenrique.anguita@urjc.es
Recibido el 27/10/2018

Revisión del 14/01 al 20/05/2019

Publicación 26/06/2019

\section{Resumen}

La configuración de la educación como un derecho y un valor en la sociedad actual está relacionada con la creación y desarrollo de las escuelas de primeras letras durante el Antiguo Régimen, las cuales en un principio estaban reservadas a las clases privilegiadas. Los planteamientos de los intelectuales ilustrados permitieron que las autoridades públicas detectaran la necesidad de mejorar la educación elemental, y de crear más y mejores escuelas de primeras letras, incluso para las clases más populares. Esta política se convirtió en uno de los principales objetivos educativos de Carlos III, quien, además, apostó por incentivar la presencia de las niñas en las escuelas de primeras letras mediante la aprobación del Reglamento para el establecimiento de escuelas gratuitas para niñas de 1783.

Palabras clave: Educación, primeras letras, Ilustración, escuelas, niñas, Carlos III. 


\begin{abstract}
The configuration of education as a right and value in today's society is related to the creation and development of primary schools during the Ancient Régime, which were originally reserved for the privileged classes. The approaches of enlightened intellectuals allowed public authorities to detect the need to improve elementary education, and to create more and better schools of the so called "first letters", even for the most popular classes. This policy became one of the main educational objectives of Carlos III, who also opted to encourage the presence of girls in schools of first letters through the adoption of the Regulation for the establishment of free schools for girls in 1783.
\end{abstract}

Keywords: Education, first letters, Enlightenment, schools, girls, Charles III of Spain 


\section{Introducción}

La educación se ha constituido, en la sociedad actual, como uno de los derechos fundamentales que toda persona debe disfrutar, ya que es una de las principales herramientas con la que los ciudadanos pueden desarrollarse a nivel, tanto personal como profesional. No obstante la preocupación por garantizar un sistema educativo de calidad, no siempre ha estado presente en el ideario político de los diferentes gobiernos. El desarrollo educativo de las personas es determinante tanto para el avance de los propios individuos como para el progreso de cualquier Estado, principalmente en las primeras etapas del aprendizaje, por lo que actualmente se debe garantizar la enseñanza en las edades más tempranas, ofreciendo un modelo eficaz de educación elemental o primaria. Para llegar a comprender mejor cómo se han configurado los vigentes modelos de educación primaria, hemos de hacer referencia a algunos de los principales hitos histórico-normativos que han contribuido en la gestación y desarrollo de la educación elemental o de primeras letras.

Poco a poco, la educación empezó a estar cada vez más presente, aunque en principio estaba al alcance de las clases más privilegiadas. Según establece Puelles, durante los siglos XVI y XVII la literatura política se centraba principalmente en garantizar la educación del príncipe (Puelles Benítez, 2010, p. 37). La crisis económica de España durante el siglo XVII tuvo su repercusión en el ámbito educativo: por un lado, según sostiene Monterrubio, en este periodo la Iglesia tuvo una presencia notable en el ámbito educativo, debido principalmente a tres motivaciones: en primer lugar, el peligro que suponía el protestantismo; en segundo lugar, la actividad de la compañía de Jesús; y finalmente, la presión ejercida por las altas autoridades de la Iglesia para tener mayor control sobre la educación española. Por otro lado, la crisis del siglo XVII supuso que las escuelas de primeras letras dejaran de ser dirigidas por los municipios $y$, en cierto modo, se privatizaran, puesto que estas instituciones empezarían a ser dirigidas por maestros privados que cobraban a los alumnos por los servicios educativos prestados, aunque al menos los municipios tendrían un control sobre 
el desarrollo y funcionamiento de las escuelas de primeras letras (Monterrubio 2006, pp. 108-110).

Desde el siglo XVIII, los intelectuales ilustrados españoles empezaron a considerar la educación del pueblo como un objetivo prioritario (Puelles 2010, p. 37), lo que permitió que poco a poco se fueran creando más escuelas de primeras letras a las que podrían acceder niños y niñas de todos los estratos sociales, incluso de los más humildes. Según recuerda Puelles, como la sociedad de la España ilustrada era estamental, la educación no tenía un carácter nacional y, además, estaba controlada por la Iglesia. A pesar de que la educación elemental existía, no estaba muy desarrollada, de modo que los nobles educaban a sus hijos en las primeras letras con la ayuda de leccionistas y preceptores. En el ámbito público, los ayuntamientos tenían pocas escuelas primarias, dirigidas por maestros mal pagados e ignorantes. Asimismo, la Iglesia tenía escuelas monásticas anejas a los conventos, que se encontraban fuera del control de la monarquía, en las que se educaban a los hijos de los campesinos (Puelles Benítez, 2010, p. 31).
Además, sostiene que los ilustrados españoles creían que la educación básica o elemental española era mediocre y escasa. En este sentido, fueron surgiendo planteamientos de intelectuales, que reclamaban una mejora de la educación de las primeras letras: Meléndez Valdés declaraba: "Lloremos sobre la inocente niñez, esperanza naciente del Estado, en la infeliz educación que ahora recibe". Campomanes manifestaba en su "Discurso sobre la educación popular" la necesidad de garantizar una educación general para todas las personas; este planteamiento se mantuvo posteriormente con Cabarrús en sus "Cartas a Jovellanos", que reiteraba la necesidad de que la educación primaria se extendiera a todos los ciudadanos, grandes y pequeños, ricos y pobres; asimismo, Jovellanos en sus "Bases para la formación de un plan general de instrucción pública"y en otras de sus obras planteaba el debate sobre la gratuidad de la enseñanza, puesto que opinaba que para formar a buenos ciudadanos se debía ofrecer una enseñanza gratuita (Puelles , 2010, pp. 38-39).

Los planteamientos de estos intelectuales sirvieron para que la educación fuera potenciada durante el siglo XVIII, 
principalmente durante el reinado de Carlos III, periodo en el que se ofreció una visión utilitarista de la educación; de modo que esta quedaría muy vinculada al desarrollo económico del país. Según manifiesta Negrín, las autoridades de la Ilustración plantearon "el desarrollo y modernización de España y para ello necesita reformar el sistema educativo tradicional, controlado hasta entonces por la Iglesia católica, y adaptarlo a las nuevas corrientes científicas, tecnológicas e ideológicas. Surge así una preocupación creciente por la educación de todas las clases sociales, incluyendo a grupos tradicionalmente marginados como las mujeres, los habitantes de los campos y los trabajadores de las ciudades, con una finalidad mezcla de utilitarismo, altruismo y humanismo..." (Negrín Fajardo, 2006, p. 187). Además, se observó un proceso de secularización en el ámbito educativo durante los reinados de Carlos III y Carlos IV, principalmente en torno a las Universidades, si bien es cierto que también se adoptaron normas y acciones que tendían a la secularización educativa en relación con las escuelas de primeras letras, de latinidad y retórica (Redondo, 2010, p. 496).
A continuación, vamos a mostrar cómo fue el origen y evolución de las escuelas de primeras letras, haciendo hincapié en la política educativa de los niños y niñas durante el reinado de Carlos III. Asimismo, se hará una especial referencia al fomento de la educación de las niñas, principalmente mediante el apoyo normativo tras la aprobación del Reglamento adoptado en Aranjuez el 11 de mayo de 1783 para el establecimiento de escuelas gratuitas en los barrios de Madrid con el fin de ofrecer una buena educación a las niñas. 


\section{Evolución de las escuelas de primeras letras y su impulso por Carlos III}

Para referirnos al origen más remoto de las escuelas de primeras letras quizás debemos remontarnos a la educación infantil de la antigua Grecia, y en concreto, a la conocida como paideia griega, organizada en base a un sistema "encíclico" o "enciclopédico (la enkyklios paideia), que a su vez se estructuraba en tres niveles. El primer nivel, denominado básico o elemental, ofrecía una formación poético musical, donde primaba la enseñanza de la lectura y memorización de textos, la escritura, el cálculo, así como la enseñanza musical. La persona encargada de ofrecer esta enseñanza era el gramatistés o "gramatista", conocido como maestro de primeras letras, y el kitharistés o "citarista", conocido como maestro de música (Redondo \& Laspalas, 2010, p. 168). Posteriormente, los modelos educativos fueron evolucionando, y en la Europa medieval, dentro de la educación popular, existían tres grados de enseñanza, que se constituían por el aprendizaje de las primeras letras, el aprendizaje de la gramática elemental y el aprendizaje de la gramática superior. Asimismo, en el medievo se desarrolló la educación gremial, orientada principalmente a preparar profesionalmente a los jóvenes para que pudieran desarrollar una actividad laboral (Laspalas, 2010, p. 288).

El modelo de las escuelas de primeras letras se asentó en el periodo comprendido desde finales del siglo XV hasta el siglo XVIII, y se basó en la creación de instituciones en las que se formaba en conocimientos básicos $y$, como apunta Ruiz, se enseñaba a los niños y niñas los principios y valores de las doctrinas cristianas, una iniciación en la lectura, el conocimiento de las cuatro cuentas aritméticas, y la enseñanza de la escritura mediante muestras y modelos (Ruiz, 2002, p. 104). A estas disciplinas, Nava añade que a los niños también se les ofrecía una enseñanza que permitiera su adecuada integración en la sociedad (Nava, 2001, p. 194). Además, Monterrubio sostiene que esta educación elemental también acabaría estando dirigida a las niñas, quienes debían aprender a desarrollar labores domésticas, coser, bordar y el modo en el que se debía dirigir una casa (Monterrubio, 2006, p. 110).

Atendiendo a los estudios realizados por Nava, el desarrollo y crecimiento de las 
escuelas de primera letras vino motivado principalmente por las siguientes causas: en primer lugar, por el incremento de la importancia de la cultura escrita en el ámbito laboral, constituyendo una vía para que la población pudiera ascender socialmente; en segundo lugar, la estrategia de la Iglesia católica y protestante de utilizar "la escuela como arma ideológica"; y en tercer lugar, la nueva visión que desde un punto de vista pedagógico y cultural se ofrecía acerca de la educación, vinculada a la infancia y la familia (Nava , 2001, pp. 184-185).

Durante el siglo XVI, las Cortes de Castilla mostraron cierta preocupación por la educación de la población infantil, puesto que en 1576 hicieron una petición a Felipe II en virtud de la cual las Justicias de diferentes territorios proponían la creación de escuelas para los niños. Ante este tipo de demandas, fueron apareciendo un conjunto de centros de primeras letras en España, entre los que se destacaban escuelas municipales, escuelas parroquiales, colegios de religiosos, escuelas privadas de primeras letras y centros de educación especial en los niveles primarios (Delgado, 1993, pp. 178-181). A pesar del aumento del interés por la educación durante el siglo
XVI, en el siglo XVII todavía no se percibió la relevancia de las escuelas de primeras letras porque las autoridades entendían que si los niños ingresaban en este tipo de instituciones se podrían descuidar las escuelas de oficios y trabajos artesanos, lo que podría repercutir negativamente en la economía del país (Delgado, 1993, p. 498).

En un primer momento, mientras las enseñanzas de primeras letras seimpartían en colegios vinculados a la Iglesia y en escuelas de gramática municipales, como preparación previa a los estudios de latinidad, las autoridades públicas no mostraron una gran preocupación por la educación primaria. Sin embargo, desde el siglo XVIII se percibieron los altos índices de analfabetismo como un grave problema que impedía el crecimiento y desarrollo del país, por lo que el Estado decidió desarrollar e implementar medidas dirigidas a mejorar la preparación de los maestros y a crear más escuelas. Según Capitán, esta circunstancia propició que bajo el reinado de Felipe $\mathrm{V}$ se aprobara la normativa que regulaba las escuelas de primeras letras, como fue la Real Cedula de 1 de septiembre de 1743, que otorgaba a los maestros de primeras letras, aprobados por la Hermandad de 
San Casiano, las mismas prerrogativas y exenciones que a los maestros de artes liberales o Humanidades (Capitán, 2002, pp. 187-188). Asimismo, durante su reinado se dio importancia a la formación e instrucción en diferentes ámbitos laborales relacionados con la agricultura, la industria, el comercio o la navegación, y se fomentó la creación de fábricasescuelas, en las cuales la educación se basaba en el traslado de las técnicas y metodologías de trabajo por diversos técnicos especializados (Elipe , 2004, 16).

En los inicios del reinado de Carlos III, la educación primaria apenas estaba desarrollada y en torno a 1760 existían muy pocas escuelas elementales en España (Desdevises du Dezert, Lorenzo y González , 1989, p. 749). Sin embargo, el monarca decidió apostar por la educación, por lo que las escuelas de primeras letras aumentaron en número y al mismo tiempo mejoraron su calidad, puesto que se decidió adoptar políticas que permitieran formar a niños y favorecer la alfabetización de las mujeres (Negrín Fajardo, 2006, p. 187). En definitiva, Carlos III decidió que uno de los grandes objetivos de su política educativa fuera la promoción de las escuelas de primeras letras, y para lograrlo, se introdujo la utilización de libros, con los que se pretendía alcanzar un doble objetivo: por un lado, los chicos aprendían a leer y, por otro lado, se les inculcaba los valores de "verdadera piedad, honor y hombría de bien" (Capitán , 2002, p. 181)

Fruto de las políticas secularizadoras y como una de las consecuencias de la expulsión de España de la Compañía de Jesús en 1767, ese mismo año se aprobó una Real Provisión el 5 de octubre, en la que se instaba a subrogar la educación de primeras letras, latinidad y retórica en maestros seculares (Redondo García, 2010, p. 496). Tan solo un año más tarde se aprobó la Real Cédula de 14 de agosto de 1768 que instituía la creación de "casas para la educación de los niños", en las que maestros seculares impartían clases de primeras letras, gramática, retórica aritmética, geometría y otras artes y además insistía en la implantación de casas de enseñanza para niñas en las que les inculcaban "principios y obligaciones de la vida civil y cristiana y enseñándoles las habilidades propias del sexo" (Capitán , 2002, p. 190).

Continuando la mejora de la educación de primeras letras, el monarca español propició la aprobación de la Real Provisión 
de 11 de julio de 1771, que exigía las condiciones necesarias para poder ejercer el magisterio de primeras letras. Con esta norma el monarca trató de imponer más celo y control en la educación elemental, mediante la inclusión de una serie de requisitos que los candidatos a maestros debían reunir para ejercer la profesión. Siguiendo a Desdevises, "el candidato a maestro debía ser cristiano viejo, de nacimiento legítimo, de buena vida y costumbres, y facilitar un certificado del juez eclesiástico demostrando que había sido interrogado sobre la doctrina cristiana. Dos examinadores y dos comisarios del Ayuntamiento le hacían pasar un examen de lectura, de escritura y de cálculo ante notario. Enviaba muestras de su escritura y de sus composiciones de cálculo a la Congregación de San Casiano de Madrid, y, según el informe de esta comisión, el Consejo de Castilla le concedía el derecho de enseñar" (Desdevises du Dezert, Lorenzo y González Enciso, 1989, p. 751).

En la década de los 80 la proliferación de la normativa educativa incrementó, destacándose la Real Provisión de 22 de diciembre de 1780, que ordenaba la creación del Colegio Académico del Noble Arte de Primeras Letras con el fin de conseguir el adelantamiento y perfección del arte de primeras letras, y extinguía la antigua Congregación de San Casiano (Capitán , 2002, p. 188). De igual manera, 1783 fue un año muy importante, puesto que se impulsó con decisión la educación de las niñas con la aprobación de la Real Cédula de 11 de mayo de 1783, en virtud de la cual se planteaba la creación de escuelas gratuitas para niñas en Madrid (Capitán , 2002, pp. 190). En base a esta norma, se instaba a la creación de 32 escuelas gratuitas para chicas en Madrid, y se fomentaba la expansión de estas medidas por el resto del reino. (Desdevises du Dezert, Lorenzo y González , 1989, p. 753). Finalmente, en los últimos momentos de su reinado llegó a aprobar más normativa educativa como fue la Real Orden de 20 de julio de 1788 sobre la creación de Escuelas Reales en Madrid. 


\section{Especial referencia} al Reglamento para el establecimiento de escuelas gratuitas para niñas de 1783

En referencia a la enseñanza de las primeras letras, en la segunda mitad del siglo XVIII se quiso potenciar la educación de las niñas, tal y como recuerda Negrín: "Al mismo tiempo, se produce una mayor preocupación por la alfabetización de las mujeres dentro de un planteamiento más amplio de transformación económica y fomento de la producción manufacturera" (Negrín, 2006, p. 187). Por todo ello, Carlos III mostró su voluntad de potenciar la educación primaria de las niñas y, para ello, hemos de aludir al Reglamento para el establecimiento de escuelas gratuitas en los barrios de Madrid para que se diera una buena educación a las niñas, adoptada en Aranjuez el 11 de mayo de $1783^{1}$. El origen y motivación de esta norma se encuentra en el éxito cosechado tras la creación de una escuela gratuita para la educación de niñas pobres del barrio de Mira el Río de Madrid:

"...Sabed, que con motivo de los buenos efectos que se han experimentado de una Escuela gratuita para la educacion de Niñas pobres del Barrio de Mira-el-Rio de Madrid, debido al zelo y actividad de los Individuos de la Diputacion de Caridad del mismo Barrio, que la promovió y estableció con aprobacion del mi Consejo, mandé prevenir á este en Real Orden de diez y siete de Octubre del próximo pasado me informase lo que se le ofreciese y pareciese sobre las varias providencias que uno de los mismos Diputados me propuso, con el fin de que, á imitación de la del citado Barrio de Mira-el-Rio, se estableciesen iguales Escuelas en los demas de Madrid..."2.

Se trata de un reglamento breve, que contaba con tan solo once artículos, y que trataba diferentes aspectos relativos a la educación primaria de las niñas en la ciudad de Madrid: fin y objeto de los establecimientos (artículo 1); número de maestras y discípulas (artículo 2); admisión de las maestras (artículo 3); comisionados (artículo 4); enseñanza (artículo 5); escuelas (artículo 6); examen de las maestras (artículo 7); algunas advertencias (artículo 8); horario de la escuela (artículo 9); emolumentos de las maestras (artículo 10); niñas que aprenden a leer (artículo 11). 
Según se recogía en el Reglamento, el principal objetivo de estas escuelas era "fomentar con transparencia á todo el Reino, la buena educacion de las jóvenes en los rudimentos de la Fe Católica, en las reglas del bien obrar, en el exercicio de las virtudes y en las labores propias del sexo, dirigiendo á las Niñas desde su infancia en los primeros pasos de su inteligencia, hasta que se proporcionen para hacer progresos en las virtudes, en el manejo de las casas, y en las labores que las corresponden, como que es la raíz fundamental de la conservación y aumento de la Religion, y el tramo que mas interesa á la policía y gobierno económico del Estado. En esta instrucción y adelantamiento logra la Causa pública la utilidad mas singular, prescindiendo de otras que son bien notorias, porque imprimiendo en las jóvenes los principios de la Religion, las buenas inclinaciones y hábitos virtuosos, al mismo tiempo que se instruye en la destreza de sus labores, no sólo se consigue criar jóvenes aplicadas, sinó que las asegura y vincula para la posteridad"s.

Por tanto, en base a este objetivo, Carlos III entendía que el medio más idóneo para formar a las niñas madrileñas fue la creación de establecimientos en los que las maestras educaran a sus discípulas. En este sentido, para lograr este objetivo se otorgó mucha relevancia a la selección de las maestras, por lo que las diputaciones de barrio debían velar "con atencion así sobre la elección de las que han de tener este cuidado, como sobre el cumplimiento de las obligaciones que se las van á imponer en este Reglamento, examinando con rigor,

\footnotetext{
${ }^{1}$ Real Cedula de S.M. y señores del Consejo, por la cual se manda observar en Madrid el Reglamento formado para el establecimiento de Escuelas gratuitas en los Barrios de él, en que se dé educación a las niñas, extendiéndose a las capitales, ciudades y villas populosas de estos reinos en lo que sea compatible con la proporción y circunstancias de cada una, y lo demás que se expresa 1783. Archivo de la Villa de Madrid (A.V.M.). Archivo de Secretaría, Tomo 41 (2-162-139).

${ }^{2}$ Ibídem, Preámbulo.
} 
no solamente la habilidad y suficiencia, sinó principalmente su buen porte y el que gobiernen con zelo sus Escuelas"4.

En cuanto a las maestras, se establecía que por el momento únicamente serían seleccionadas unas 32 maestras, las cuales debían ser admitidas y nombradas previa presentación y evaluación de un riguroso informe que valorara sus habilidades y circunstancias. Se advertía que no podía enseñar ni ejercer las funciones de maestra pública ninguna persona que no hubiera sido admitida y aprobada por las correspondientes diputaciones de barrio. Asimismo, las maestras contarían con la colaboración de una de sus discípulas, que haría de "Ayudanta", la cual sería elegida entre aquellas que reunieran las habilidades necesarias y tuvieran buenas costumbres 5 .

En la elección de las nuevas maestras tenían preferencia aquellas que ya estuvieran en la corte, salvo que no reunieran las habilidades requeridas y no cumplieran las buenas costumbres. Para presentar la candidatura como maestra, las mujeres debían aportar un memorial a las diputaciones de barrio en donde destacarían sus habilidades y conducta, siendo seleccionada finalmente la candidata que la diputación considerara más "digna"

Para acceder al puesto de maestra, las candidatas al puesto debían reunir una serie de condiciones: en primer lugar, las maestras eran examinadas con rigor en la doctrina cristiana o en su defecto debían aportar un certificado emitido por un párroco que acreditara su doctrina mencionada; en segundo lugar, debían realizar un examen de labores "delante de las otras Maestras por el turno que establezcan las Diputaciones para que no haya favor y se reconozca en todas el grado de habilidad que tuviesen. Se las preguntaráel mododehacercadalaboryel método de enseñarla, y presentarán algún trabajo de lo que deben enseñar, hecho de su mano; y así executado, se preferirá siempre á la de mejores costumbres en concurso de igual habilidad..."; en tercer lugar, las diputaciones de barrio debían tomar informes "de su buena vida y costumbres, y de las de sus maridos, si fuesen casadas"

En cuanto a las retribuciones de las maestras, se establecía que recibirían una contribución económica de los padres que tuvieran algo de solvencia 
económica, declarando expresamente que las niñas pobres estudiarían de forma gratuita y con la misma calidad educativa: "Las Niñas, cuyos padres tuviesen con que pagar su enseñanza, contribuirán á las Maestras con la moderada cantidad que hasta ahora han acostumbrado, ó tratarán con sus padres ó tutores el honorario que les deban dar, pero á las pobres se las enseñará de valde con el mismo cuidado que á las que pagan, pues así lo exige la caridad y la buena policía...". No obstante, la Junta General de Caridad trataría de ayudar económicamente a las diputaciones de barrio para que consiguieran 50 pesos anuales, además de las contribuciones que pagaran los padres de las niñas más pudientes ${ }^{8}$.

Respecto a las materias que las niñas aprendían en estas escuelas, en primer lugar, recibían una formación religiosa: "Lo primero que enseñarán las Maestras á las Niñas serán las Oraciones de la Iglesia, la Doctrina Christiana por el método del catecismo...". Además, las inculcarían principios y valores como "las máximas de pudor y de buenas costumbres", y al mismo tiempo, las obligaban a que fueran limpias y aseadas a la escuela, y que allí se mantuvieran "con modestia y quietud". Mientras las niñas estuvieran en la escuela se debían "ocuparen suslabores, cada una en la que la corresponda", bajo la vigilancia de las maestras que debían "cuidar tanto del aprovechamiento, como de que unas no perturben á otras, y de que en todas se observe buen orden". Las maestras primero debían enseñar a las discípulas las labores más sencillas y avanzar más tarde con las más complicadas: "...empezando por las más fáciles, como Faxa, Calceta, punto de Red, Dechado, Dobladillo, Costura, siguiendo después á coser mas fino, bordar, hacer Encages, y en otros ratos que acomodará la Maestra según su inteligencia, hacer Cofias ó Redecillas, sus Borlas, Bolsillos..." Sin embargo, a pesar de que el principal
${ }^{3}$ Ibídem, Art. 1.
${ }^{6}$ Ibídem, Art. 3.
${ }^{4}$ Ibídem, Art. 1.
${ }^{7}$ Ibídem, Art. 7.
${ }^{5}$ Ibídem, Art. 2.
${ }^{8}$ Ibídem, Art. 10. 
objeto de estas escuelas era la enseñanza de labores manuales, si alguna de las muchachas tenía interés en aprender a leer, la maestra tenía la obligación de enseñarla ${ }^{10}$

En términos generales, durante la enseñanza la comunicación entre las maestras y las niñas debía ser didáctica, y aquellas debían tener "un estilo claro y sencillo en la explicación de la enseñanza é instruccion que dieren á sus Discípulas". Asimismo, debían vigilar el lenguaje de sus discípulas: "...no permitirán á éstas usar palabras indecentes, equívocas, ni de aquéllas que se dicten propias de las majas"11.

Atendiendo al funcionamiento de las escuelas, se pueden precisar algunas cuestiones: en primer lugar, todas las escuelas de la corte debían ser examinadas y aprobadas por los comisarios de las diputaciones de barrio; en segundo lugar, las maestras no podían solicitar la concurrencia de niñas de otras escuelas, ni admitir en la suya a niñas que hubieran asistido a otras, sin que previamente no se hubiera informado sobre el motivo que fundamentara esta decisión; además, las maestras tenían prohibido dejar de asistir a sus escuelas, salvo en supuestos de enfermedad, que serían sustituidas por las "Ayudantas"12; respecto al horario, las maestras y ayudantas debían asistir a la escuela e impartir la enseñanza de las niñas cuatro horas por la mañana y otras cuatro por la tarde, variando según las estaciones del año, pero sin poder disminuir las horas ${ }^{13}$; Además, Carlos III ordenó que, tras el éxito de estas escuelas, se implantaran en las demás capitales, ciudades y villas del reino.

\footnotetext{
${ }^{9}$ Ibídem, Art. 5.

12 Ibídem, Art. 6.

${ }^{10}$ Ibídem, Art. 11.

${ }^{13}$ Ibídem, Art. 9.

${ }^{11}$ Ibídem, Art. 8.
} 


\section{Conclusiones}

Es importante volver a recordar la constatada importancia que ha ido acaparando la educación a lo largo de la historia, tanto fuera como dentro de España. Desde sus orígenes más remotos en la Edad Antigua hasta el reinado de Carlos III, durante la Edad Moderna, en términos generales, se ha observado un proceso de transformación en la educación, principalmente desde dos puntos de vista: en primer lugar, en tiempos más remotos el acceso a la educación únicamente estaba al alcance de las clases sociales más privilegiadas, hasta que de forma paulatina, los colectivos más humildes de la población fueron accediendo a la enseñanza; en segundo lugar, en un principio la educación estuvo muy marcada por la influencia de la religión, no obstante, con el paso de los años este enmarcado carácter religioso dejó de tener tanta importancia, y se potenció la educación pública.

Con la llegada de los borbones a España se apostó por la educación, y en concreto, por la educación elemental y las escuelas de primeras letras, en las que se formaba a los niños y niñas y recibían formación religiosa inculcándoles los valores y principios de las doctrinas cristianas. Además, les iniciaban en la lectura, les enseñaban las principales reglas aritméticas, y perfeccionaban su escritura. A pesar del interés por potenciar estas instituciones educativas, todavía las autoridades políticas no eran conscientes de la importancia real que tenía la educación para el progreso económico y social del Estado.

Algunos intelectuales ilustrados españoles como Meléndez Valdés, Campomanes o Cabarrús observaron la importancia de potenciar la educación y las escuelas de primeras letras. Estos planteamientos fueron secundados por Carlos III, quien llegó con el objetivo de modernizar las instituciones españolas, y se propuso impulsar las artes, la cultura y, por supuesto, la educación. En este sentido, desarrolló políticas educativas para potenciar la educación no solamente para las clases privilegiadas, sino también para los niños y niñas procedentes de las familias más pobres. 
Durante su reinado, no solamente produjo un gran avance intelectual, y proliferó abundante normativa que posteriormente en pleno siglo XVIII, potenciaba la educación elemental y la durante el reinado de Carlos III se creación de escuelas de primeras letras, sino que además trató de impulsar aún más la educación de las niñas, por lo que promovió la aprobación del famoso Reglamento de 1783 para el establecimiento de escuelas gratuitas trató de reformar y mejorar el modelo de educación pública, llegándose a conseguir grandes avances, únicamente frenados tras la invasión francesa en España (Desdevises du Dezert, Lorenzo y para las niñas madrileñas. Esta norma trató de regular de una forma global la educación primaria de las niñas, para que recibieran una educación gratuita, eficaz y de calidad.

Asimismo, el Reglamento planteaba la creación de escuelas gratuitas para niñas en los diferentes barrios de Madrid, y para garantizar un buen servicio educativo, incorporaba una serie de requisitos exhaustivos para la selección de las futuras maestras, los exámenes que debían superar, y las retribuciones que tenían que recibir. Además, se contemplaban otros aspectos relevantes relativos al funcionamiento de las escuelas, como la enseñanza que se ofrecía, las materias y habilidades que se aprendían o los horarios de las clases.

En definitiva, en términos generales, en la España de los siglos XVI y XVII se 


\section{Referencias}

Capitán Díaz, A. (2002). Breve historia de la educación en España. Madrid: Alianza.

Delgado Criado, B. (1993). Historia de la educación en España y América. La educación en la España moderna (Siglos XVI-XVIII). Madrid: SM.

Desdevises du Dezert, G., Lorenzo González, A., y González , A. (1989). La España del Antiguo Régimen. Madrid: Fundación Universitaria Española.

Elipe , J. (2004). Historia Constitucional del Derecho a la Educación en España. Valencia: Nomos.

Laspalas, J. (2010). La educación durante la edad media. En E. Redondo García, Introducción a la Historia de la Educación (págs. 251-354). Barcelona: Ariel.

Monterrubio , Á. (2006). Educación y política del Barroco. En O. Negrín Fajardo, Historia de la educación española (págs. 103-140). Madrid: Universidad Nacional de Educación a Distancia.

Nava, T. (2001). La escuela y su mundo: concepto y transmisión de los saberes elementales en los siglos moderno. En Burke, J Martín Martín, T. Nava, y J.L. Guereña, Educación y transmisión de conocimientos en la historia (págs. 183-209). Salamanca: Universidad de Salamanca.

Negrín , O. (2006). Realizaciones y proyectos ilustrados. En O. Negrín Fajardo, Historia de la Educación española (págs. 185-226). Madrid: Universidad Nacional de Educación a Distancia.

Puelles, M. (2010). Educación e ideología en la España contemporánea. Madrid: Tecnos.

Redondo García, E. (2010). La educación del siglo XVIII. En E. Redondo García, Introducción a la Historia de la Educación (págs. 467-519). Barcelona: Ariel.

Redondo, E., y Laspalas, J. (2010). La paideia griega. En E. Redondo García, Introducción a la Historia de la Educación (págs. 121-176). Barcelona: Ariel.

Ruiz , J. (2002). El sistema educativo español: de las Cortes de Cádiz a la Ley 
Moyano. En A. Tiana Ferrer, G. Ossenbach

Sauter, y F. Sanz Fernández, Historia de la educación (Edad Contemporánea) (págs. 91-116). Madrid: Universidad Nacional de Educación a Distancia.

\section{Consultas de archivo}

Archivo de la Villa de Madrid (A.V.M.). Archivo de Secretaría, Tomo 41 (2-162139).

\section{Para citar este artículo:}

$$
\text { Anguita, E (2019). }
$$

Evolución de las escuelas de primeras letras y su impulso por Carlos III. Especial referencia al reglamento para el establecimiento de escuelas gratuitas para niñas de 1783 .

Revista Educa UMCH, 13(1). https://doi.org/10.35756/educaumch.v7i13.92 